\title{
ON THE UNIQUENESS OF MEROMORPHIC FUNCTIONS SHARING THREE-POINT SETS IM
}

\author{
TAKAhiro Kongo ${ }^{1}$ And Manabu Shirosaki ${ }^{2}$ \\ ${ }^{1}$ Department of Mathematical Sciences, School of Engineering, Osaka \\ Prefecture University, Sakai 599-8531, Japan, i.l.my.llife@gmail.com \\ ${ }^{2}$ Department of Mathematical Sciences, School of Sciences, Osaka Prefecture \\ University, Sakai 599-8531, Japan, mshiro@ms.osakafu-u.ac.jp
}

\begin{abstract}
We show that if two meromorphic functions on the complex plane sharing some three-point sets IM, then they are indentical under some conditions.

Key words and phrases: Uniqueness theorem, Sharing sets, Nevanlinna theory
\end{abstract}

\section{INTRODUCTION AND THE MAIN RESULTS}

For nonconstant meromorphic functions $f$ and $g$ on $\mathbb{C}$ and a finite set $S$ in $\overline{\mathbb{C}}=\mathbb{C} \cup\{\infty\}$, we say that $f$ and $g$ share $S \mathrm{CM}$ (counting multiplicities) if $f^{-1}(S)=g^{-1}(S)$ and if for each $z_{0} \in f^{-1}(S)$ two functions $f-f\left(z_{0}\right)$ and $g-g\left(z_{0}\right)$ have the same multiplicity of zero at $z_{0}$, where the notations $f-\infty$ and $g-\infty$ mean $1 / f$ and $1 / g$, respectively. Also, if $f^{-1}(S)=g^{-1}(S)$, then we say that $f$ and $g$ share $S$ IM (ignoring multiplicities). In particular if $S$ is a one-point set $\{a\}$, then we say also that $f$ and $g$ share $a$ CM or IM. The notion of meromorphic functions sharing sets is introduced by Gross in [1].

In [4] and [5], R. Nevanlinna showed the following two theorems:

Theorem 1.1. Let $f$ and $g$ be two distinct nonconstant meromorphic functions on $\mathbb{C}$ and let $a_{1}, \cdots, a_{4}$ be four distinct points in $\overline{\mathbb{C}}$. If $f$ and $g$ share $a_{1}, \cdots, a_{4}$ $C M$, then $f$ is a Möbius transform of $g$, i.e. $f=(a g+b) /(c g+d)$ for some complex numbers $a, b, c, d$ with $a d-b c \neq 0$, and there exists a permutation $\sigma$ of $\{1,2,3,4\}$ such that $a_{\sigma(3)}, a_{\sigma(4)}$ are Picard exceptional values of $f$ and $g$ and the cross ratio $\left(a_{\sigma(1)}, a_{\sigma(2)}, a_{\sigma(3)}, a_{\sigma(4)}\right)=-1$.

2020 Mathematics Subject Classification: 30D35 Received: 04-08-2019, accepted: 02-05-2020. 
Theorem 1.2. Let $f$ and $g$ be two nonconstant meromorphic functions on $\mathbb{C}$ sharing five distinct points in $\overline{\mathbb{C}} I M$, then $f=g$.

Since Nevanlinna, many researchers have been studying various versions of uniquness theorem of meromorphic functions sharing finite sets CM or IM (see, e.g., [7], [8], [11] and [12]). In particular, the second author researched meromorphic functions sharing four two-point sets CM, and also he gave the following result in [9]

Theorem 1.3. Let $S_{1}, \cdots, S_{5}$ be pairwise disjoint one-point or two-point sets in $\overline{\mathbb{C}}$. If two nonconstant meromorphic functions $f$ and $g$ on $\mathbb{C}$ share $S_{1}, \cdots, S_{5} I M$, then $f$ is a Möbius transform of $g$.

Remark. Let $T$ be the Möbius transformation of the conclusion in Theorem 1.3. By the proof of Theorem 1.3, we see that $T$ is of order 2, i.e., $T^{2}=T \circ T$ is the identity if $T$ is not the identity.

Since any Möbius transformation except the indentity has at most two fixed points and any nonconstant meromorphic function has at most two exceptional values, we have, as a corollary of Theorem 1.3,

Corollary 1.4. Let $S_{1}, \cdots, S_{5}$ be pairwise disjoint one-point or two-point sets in $\overline{\mathbb{C}}$. Assume that there is no Möbius transformation $T$ except the identity with at most two points $z$ in $\overline{\mathbb{C}}$ satisifying one of the following conditions: (i) $z \in S_{j}$ and $T(z) \notin S_{j}$ for some $j=1, \cdots, 5$; (ii) $z \notin \cup_{j=1}^{5} S_{j}$ and $T(z) \in \cup_{j=1}^{5} S_{j}$. Then two nonconstant meromorphic functions on $\mathbb{C}$ sharing $S_{1}, \cdots, S_{5}$ IM are identical.

In particular, in the case of five two-point sets we see

Theorem 1.5. Let $S_{1}, \cdots, S_{5}$ be pairwise disjoint two-point sets in $\overline{\mathbb{C}}$. Assume that there is no Möbius transformation which interchanges two elements of $S_{j}$ for distinct three $j$. Then, two nonconstant meromorphic functions on $\mathbb{C}$ sharing $S_{1}, \cdots, S_{5}$ IM are identical.

We assume that $S_{1}, S_{2}, S_{3}$ be pairwise disjoint two-point sets in $\mathbb{C}$. Let $S_{j}=\left\{\xi_{j}, \eta_{j}\right\}$ and let $P_{j}(z)=z^{2}+a_{j} z+b_{j}$, where $a_{j}=-\left(\xi_{j}+\eta_{j}\right), b_{j}=\xi_{j} \eta_{j}$. We see that the following three conditions are equivalent: (i) there exists a Möbius transformation which interchanges $\xi_{j}$ and $\eta_{j}$ for $j=1,2,3$; (ii) $\left|\begin{array}{ccc}1 & \xi_{1}+\eta_{1} & \xi_{1} \eta_{1} \\ 1 & \xi_{2}+\eta_{2} & \xi_{2} \eta_{2} \\ 1 & \xi_{3}+\eta_{3} & \xi_{3} \eta_{3}\end{array}\right|=0$; (iii) $P_{1}, P_{2}, P_{3}$ are linearly dependent over $\mathbb{C}$ (see Lemma 3.2 in [6]). 
For a finite set $S$ in $\mathbb{C}$, we define its defining polynomial $P$ as a polynomial without multiple zeros satisfying $S=\{z \in \mathbb{C}: P(z)=0\}$, then $P$ is called a defining polynomial of $S$. Therefore, we can restate Theorem 1.5 as follows:

Theorem 1.6. Let $S_{1}, \cdots, S_{5}$ be pairwise disjoint two-point sets in $\mathbb{C}$ and let $P_{j}$ be a defining polynomial of $S_{j}$. If any three of $P_{1}, \cdots, P_{5}$ are linearly independent over $\mathbb{C}$, then two nonconstant meromorphic functions on $\mathbb{C}$ sharing $S_{1}, \cdots, S_{5} I M$ are identical.

In this paper, we consider the uniqueness of meromorphic functions sharing three-point sets and one-point sets IM. The above condition (i) is geometrical, but it is hard to treat it in our problem. Therefore we adapt the condition (iii) to our problem, and about the uniqueness of meromorphic functions sharing three-point sets IM, we show

Theorem 1.7. Let $S_{1}, \cdots, S_{5}$ be pairwise disjoint three-point sets in $\mathbb{C}$ and let $P_{j}$ be a defining polynomial of $S_{j}$. If any three of $P_{1}, \cdots, P_{5}$ are linearly independent over $\mathbb{C}$, then two nonconstant meromorphic functions on $\mathbb{C}$ sharing $S_{1}, \cdots, S_{5} I M$ are identical.

We assume that the reader is familiar with the standard notations and results of the value distribution theory (see, for example, [2]). In particular, we express by $S(r, f)$ quantities such that $\lim _{r \rightarrow \infty, r \notin E} S(r, f) / T(r, f)=0$, where $E$ is a subset of $(0, \infty)$ with finite linear measure and it is variable in each case.

\section{PROOF OF THEOREM 1.7}

First, for general polynomials we show the following lemma.

Lemma 2.1. Let $P_{1}, P_{2}, P_{3}$ be three polynomials of degree $d$, where $d$ is a positive integer. Assume that $P_{j}$ has no multiple zeros and that $P_{j}$ and $P_{k}$ have no common zeros for distinct $j$ and $k$ in $\{1,2,3\}$. Moreover, assume that $P_{1}, P_{2}, P_{3}$ are linearly independent over $\mathbb{C}$. Then not all of $P_{1}\left(\xi_{j}\right) / P_{2}\left(\xi_{j}\right)(j=1, \cdots, d)$ are the same, where $\xi_{1}, \cdots, \xi_{d}$ are the zeros of $P_{3}$.

Proof. On the contrary, we assume that all of of $P_{1}\left(\xi_{j}\right) / P_{2}\left(\xi_{j}\right)(j=1, \cdots, d)$ are the same value $\lambda$ :

$$
P_{1}\left(\xi_{j}\right)-\lambda P_{2}\left(\xi_{j}\right)=0 \quad(j=1, \cdots, d) .
$$

Put $Q(z)=P_{1}(z)-\lambda P_{2}(z)$, then $Q$ has zeros $\xi_{1}, \cdots, \xi_{d}$, and hence it is a nonzero complex multiple of $P_{3}$. This implies that $P_{1}, P_{2}, P_{3}$ are linearly dependent over $\mathbb{C}$, and hence, we have finished the proof. 
We introduce the following Borel's Lemma, whose proof can be found, for example, on p.186 of [3].

Lemma 2.2. If entire functions $\alpha_{0}, \alpha_{1}, \ldots, \alpha_{n}$ without zeros satisfy

$$
\alpha_{0}+\alpha_{1}+\cdots+\alpha_{n}=0,
$$

then for each $j=0,1, \cdots, n$ there exists some $k \neq j$ such that $\alpha_{j} / \alpha_{k}$ is constant.

For the proof of Theorem 1.7 we need the following result in [10]

Theorem 2.3. Let $p$ be a non-negative integer and let $q$ be an integer not less than 2. Let $S_{1}, \cdots, S_{p}$ be one-point sets in $\mathbb{C}$ and let $S_{p+1}, \cdots, S_{p+q}$ be $d$-point sets in $\mathbb{C}$, where $d$ is an integer not less than 2. Assume that $S_{1}, \cdots, S_{p+q}$ are pairwise disjoint and that $p+q \geq 5$. If two distinct nonconstant meromorphic functions $f$ and $g$ on $\mathbb{C}$ share $S_{1}, \cdots, S_{p+q} I M$, then there exists distinct $j_{1}, j_{2}$ in $\{p+1, \cdots, p+q\}$ such that $P_{j_{1}}(f) / P_{j_{2}}(f)=P_{j_{1}}(g) / P_{j_{2}}(g)$, where $P_{j}$ are defining polynomials of $S_{j}$.

Proof. We may assume that $p \leq 4$ by Theorem 1.2 .

By the second main theorem and the first main theorem we have

$$
\begin{aligned}
& (p+d q-2) T(r, f) \leq \sum_{j=1}^{p+q} \sum_{\xi \in S_{j}} \bar{N}\left(r, \frac{1}{f-\xi}\right)+S(r, f) \\
& =\sum_{j=1}^{p+q} \sum_{\xi \in S_{j}} \bar{N}\left(r, \frac{1}{g-\xi}\right)+S(r, f) \leq(p+d q) T(r, g)+S(r, f)
\end{aligned}
$$

and, by the same way,

$$
(p+d q-2) T(r, g) \leq(p+d q) T(r, f)+S(r, g) .
$$

Hence, by (1) and (2), there is no need to distinguish $S(r, f)$ and $S(r, g)$, and so we denote them by $S(r)$.

By $\bar{N}_{E}\left(r, \frac{1}{f-\xi}\right)$ and $\bar{N}_{N}\left(r, \frac{1}{f-\xi}\right)$ we denote the counting functions which count the point $z$ such that $f(z)=\xi=g(z)$ and $f(z)=\xi \neq g(z)$ counted once, respectively, and we define $\bar{N}_{E}\left(r, \frac{1}{g-\xi}\right)$ and $\bar{N}_{N}\left(r, \frac{1}{g-\xi}\right)$ by the same way. It is easy to see that $\bar{N}_{N}\left(r, \frac{1}{f-\xi}\right)=\bar{N}_{N}\left(r, \frac{1}{g-\xi}\right)=0$ for $\xi \in S_{1} \cup \cdots \cup S_{p}$ and that

$$
\begin{aligned}
\sum_{\xi \in S_{j}} \bar{N}_{E}\left(r, \frac{1}{f-\xi}\right) & =\sum_{\xi \in S_{j}} \bar{N}_{E}\left(r, \frac{1}{g-\xi}\right), \\
\sum_{\xi \in S_{j}} \bar{N}_{N}\left(r, \frac{1}{f-\xi}\right) & =\sum_{\xi \in S_{j}} \bar{N}_{N}\left(r, \frac{1}{g-\xi}\right)
\end{aligned}
$$


for $j=p+1, \cdots, q$. Since $f-g \not \equiv 0$, we have

$$
\sum_{j=1}^{p+q} \sum_{\xi \in S_{j}} \bar{N}_{E}\left(r, \frac{1}{f-\xi}\right) \leq \bar{N}\left(r, \frac{1}{f-g}\right) \leq T(r, f)+T(r, g)+O(1),
$$

and hence

$$
\begin{aligned}
\sum_{j=p+1}^{p+q} \sum_{\xi \in S_{j}} \bar{N}_{N}\left(r, \frac{1}{f-\xi}\right) & =\sum_{j=1}^{p+q} \sum_{\xi \in S_{j}} \bar{N}\left(r, \frac{1}{f-\xi}\right)-\sum_{j=1}^{p+q} \sum_{\xi \in S_{j}} \bar{N}_{E}\left(r, \frac{1}{f-\xi}\right) \\
& \geq(p+d q-2) T(r, f)-T(r, f)-T(r, g)+S(r) \\
& =(p+d q-3) T(r, f)-T(r, g)+S(r)
\end{aligned}
$$

by using (1). By the same way and (3) we have

$$
\sum_{j=p+1}^{p+q} \sum_{\xi \in S_{j}} \bar{N}_{N}\left(r, \frac{1}{f-\xi}\right) \geq(p+d q-3) T(r, g)-T(r, f)+S(r) .
$$

Adding these two inequalities we obtain

$$
\sum_{j=p+1}^{p+q} \sum_{\xi \in S_{j}} \bar{N}_{N}\left(r, \frac{1}{f-\xi}\right) \geq \frac{1}{2}(p+d q-4)(T(r, f)+T(r, g))+S(r) .
$$

Note that $q \geq 2$. From (4) we see that there exist distinct $j_{1}$ and $j_{2}$ in $\{p+1, \cdots, q\}$ and a subset $I$ of $(0,+\infty)$ of infinite linear measure such that

$$
\frac{1}{q}(p+d q-4)(T(r, f)+T(r, g))+S(r) \leq \sum_{\xi \in S_{j_{1}} \cup S_{j_{2}}} \bar{N}_{N}\left(r, \frac{1}{f-\xi}\right)
$$

holds for $r \in I$. Put $Q(z, w)=\left(P_{j_{1}}(z) P_{j_{2}}(w)-P_{j_{1}}(w) P_{j_{2}}(z)\right) /(z-w)$ and $\Phi=$ $Q(f, g)$. Assume that $\Phi \not \equiv 0$. If $f(z), g(z) \in S_{j_{1}} \cup S_{j_{2}}$ and $f(z) \neq g(z)$, then $\Phi(z)=0$. Therefore we have

$$
\sum_{\xi \in S_{j_{1}} \cup S_{j_{2}}} \bar{N}_{N}\left(r, \frac{1}{f-\xi}\right) \leq N_{0}\left(r, \frac{1}{\Phi}\right)
$$

holds for $r \in I$, where $N_{0}\left(r, \frac{1}{\Phi}\right)$ denotes the counting functions corresponding to the zeros of $\Phi$ that are not the poles of $f$ and $g$. We see that $Q(z, w)$ is a symmetric polynomial of $z$ and $w$ and it has degree at most $d-1$ with respect to each of $z$ and $w$. By using the first fundamental theorem and the definition of countiong function and that of proximity function, we have

$$
\begin{aligned}
N_{0}\left(r, \frac{1}{\Phi}\right) & \leq N(r, Q(f, g))+m(r, Q(f, g)) \\
& \leq(d-1)(N(r, f)+N(r, g)+m(r, f)+m(r, g))+O(1) \\
& =(d-1)(T(r, f)+T(r, g))+O(1) .
\end{aligned}
$$

By connecting (5), (6) and this,

$$
\frac{1}{q}(p+d q-4)(T(r, f)+T(r, g))+S(r) \leq(d-1)(T(r, f)+T(r, g))+O(1)
$$


holds for $r \in I$. Here $I$ may be different from that in (5). We obtain $p+d q-4 \leq$ $q(d-1)$, which contradicts hypothesis $p+q \geq 5$. Therefore we conclude that $\Phi \equiv 0$, which induces that $P_{j_{2}}(f) / P_{j_{1}}(f)=P_{j_{2}}(g) / P_{j_{1}}(g)$. We have completed the proof.

Now, we start the proof of Theorem 1.7. By Theorem 2.3, we may assume that $P_{1}(f) / P_{2}(f)=P_{1}(g) / P_{2}(g)$ by rearranging indices if necessary.

Remark. Let $P_{1}, \cdots, P_{q}$ be polynomials of degree $d$, where $q$ and $d$ are integers such that $q \geq 3, d \geq 2$. Assume that each of $P_{1}, \cdots, P_{q}$ has no multiple zeros and that any two of them have no common zeros. Moreover, we assume that $P_{1}(f) / P_{2}(f)=P_{1}(g) / P_{2}(g)$ for nonconstant meromorphic functions $f$ and $g$ on $\mathbb{C}$ and that $f$ and $g$ share $S_{3}, \cdots, S_{q}$ IM. If for each $j=3, \cdots, q$ the $d$ values $P_{1}\left(\xi_{j k}\right) / P_{2}\left(\xi_{j k}\right)(k=1, \cdots, d)$ are distinct for each zero $\xi_{j k}$ of $P_{j}$, then $f$ and $g$ share $\xi_{j k}$ IM. In this case, by Theorem 1.2 , we see that $f=g$ if $d(q-2) \geq 5$. However, the hypothesis that for each $j=3, \cdots, q$ the $d$ values $P_{1}\left(\xi_{j k}\right) / P_{2}\left(\xi_{j k}\right)(k=$ $1, \cdots, d)$ are distinct is too strong, and we seek another condition about $P_{j}$ for the uniqueness of meromorphic functions.

Again, we return to the proof. Let $S_{j}=\left\{\xi_{j}, \eta_{j}, \zeta_{j}\right\}$. For $\xi, \eta \in \overline{\mathbb{C}}$, we put $E(\xi, \eta)=\{z \in \mathbb{C}:(f(z), g(z))=(\xi, \eta)$ or $(\eta, \xi)\}$. We separate $S_{3}, S_{4}, S_{5}$ into two types: (A) some of $E\left(\xi_{j}, \eta_{j}\right), E\left(\xi_{j}, \zeta_{j}\right), E\left(\eta_{j}, \zeta_{j}\right)$ are not empty; (B) all of $E\left(\xi_{j}, \eta_{j}\right), E\left(\xi_{j}, \zeta_{j}\right), E\left(\eta_{j}, \zeta_{j}\right)$ are empty. Then we consider three cases: (I) at least two of $S_{3}, S_{4}, S_{5}$ are of type (B); (II) one of $S_{3}, S_{4}, S_{5}$ is of type (B) and the others are of type (A); (III) all of $S_{3}, S_{4}, S_{5}$ are of type (A).

The case (I). At least two of $S_{3}, S_{4}, S_{5}$ are of type (B). We may assume that $S_{4}, S_{5}$ are of type (B). Then $f$ and $g$ share $\xi_{j}, \eta_{j}, \zeta_{j}$ IM for $j=4,5$. By Theorem 1.2 , we get $f=g$.

The case (II). One of $S_{3}, S_{4}, S_{5}$ is of type (B) and the others are of type (A). We may assume that $S_{3}$ is of type (B) and $S_{4}$ and $S_{5}$ are of type (A). Moreover, we may assume that $E\left(\xi_{j}, \eta_{j}\right) \neq \emptyset$ for $j=4,5$. Then, for $j=4,5$, we have $P_{1}\left(\xi_{j}\right) / P_{2}\left(\xi_{j}\right)=P_{1}\left(\eta_{j}\right) / P_{2}\left(\eta_{j}\right)$, and by assumption and Lemma 2.1 we have $E\left(\xi_{j}, \zeta_{j}\right)=E\left(\eta_{j}, \zeta_{j}\right)=\emptyset$, which implies that $f$ and $g$ share $\left\{\xi_{j}, \eta_{j}\right\}$ and $\left\{\zeta_{j}\right\}$ IM. Since $f$ and $g$ share also $\xi_{3}, \eta_{3}, \zeta_{3}$ IM, by using Theorem 1.2, we get $f=g$.

The case (III). All of $S_{3}, S_{4}, S_{5}$ are of type (A). We may assume that $E\left(\xi_{j}, \eta_{j}\right)$ $\neq \emptyset$ for $j=3,4,5$. Then, for $j=3,4,5$, we have $P_{1}\left(\xi_{j}\right) / P_{2}\left(\xi_{j}\right)=P_{1}\left(\eta_{j}\right) / P_{2}\left(\eta_{j}\right)$, and by assumption and Lemma 2.1 we have $E\left(\xi_{j}, \zeta_{j}\right)=E\left(\eta_{j}, \zeta_{j}\right)=\emptyset$, which implies that $f$ and $g$ share $\left\{\xi_{j}, \eta_{j}\right\}$ and $\left\{\zeta_{j}\right\}$ IM. By using Theorem 1.3, there exists a Möbius transformation $T$ such that $f=T \circ g$.

Assume that $f \not \equiv g$, and hence $T$ is not the identity. Then $T$ has at most two fixed points, and hence, we may assume that $E\left(\zeta_{5}, \zeta_{5}\right)=\emptyset$. In this case, $\zeta_{5}$ is an exceptioanl value of $f$ and $g$. Furthermore, $T$ interchanges $\xi_{j}$ and $\eta_{j}$ for at least one of $j \in\{3,4,5\}$, which are also fixed points of $T^{2}=T \circ T$. Since the fixed points of $T$ are also those of $T^{2}$, it has at least three fixed points. Therefore, $T^{2}$ is the identity. This result follows also from Remark of Theorem 1.3. 
(i) The case where $E\left(\zeta_{4}, \zeta_{4}\right)=\emptyset$ or $E\left(\zeta_{3}, \zeta_{3}\right)=\emptyset$. It is enough to consider the case where $E\left(\zeta_{4}, \zeta_{4}\right)=\emptyset$. In this case $\zeta_{4}$ and $\zeta_{5}$ are exceptional value of $f$ and $g$ and they have no more exceptional values. Therefore $E\left(\zeta_{3}, \zeta_{3}\right) \neq \emptyset$, and hence $\zeta_{3}$ is a fixed point $T$. Since $T$ has at most two fixed points we may assume that all $E\left(\xi_{1}, \xi_{1}\right), E\left(\eta_{1}, \eta_{1}\right), E\left(\zeta_{1}, \zeta_{1}\right)$ are empty. Furthermore, we may assume that $E\left(\xi_{1}, \eta_{1}\right) \neq \emptyset$ since $f^{-1}\left(S_{1}\right) \neq \emptyset$. Then $T\left(\xi_{1}\right)=\eta_{1}$ and $T\left(\eta_{1}\right)=\xi_{1}$. Since $T$ is a one-to-one mapping of $\overline{\mathbb{C}}$ onto $\overline{\mathbb{C}}, E\left(\xi_{1}, \zeta_{1}\right)=\emptyset, E\left(\eta_{1}, \zeta_{1}\right)=\emptyset$. However, this implies that $\zeta_{1}$ is an exceptional value of $f$ and $g$, which is a contradiction.

(ii) The case where $E\left(\zeta_{3}, \zeta_{3}\right) \neq \emptyset$ and $E\left(\zeta_{4}, \zeta_{4}\right) \neq \emptyset$. In this case $\zeta_{3}$ and $\zeta_{4}$ are fixed points of $T$, and it has no more fixed points, in particular, $E\left(\xi_{j}, \xi_{j}\right)=$ $E\left(\eta_{j}, \eta_{j}\right)=E\left(\zeta_{j}, \zeta_{j}\right)=\emptyset$ for $j=1,2$. Since $f$ and $g$ have an exceptional value $\zeta_{5}$, we may assume that they have no exceptional value in $S_{1}$. Hence we may assume that $E\left(\xi_{1}, \eta_{1}\right) \neq \emptyset$, and we can get a contradiction as in the case (i).

After all, we have $f=g$, and the proof is completed.

\section{MEROMORPHIC FUNCTIONS SHARING TWO THREE-POINT SETS AND THREE VALUES IM}

Let $f$ and $g$ be two meromorphic functions on $\mathbb{C}$ sharing $S_{1}, \cdots, S_{5}$ IM, where $S_{1}, S_{2}$ are three-point sets in $\mathbb{C}$ and $S_{3}, S_{4}, S_{5}$ are one-point sets in $\mathbb{C}$, and $S_{1}, \cdots, S_{5}$ are pairwise disjoint. Suppose that $f \not \equiv g$. Then, by using Theorem 2.3 , we have $P_{1}(f) / P_{2}(f)=P_{1}(g) / P_{2}(g)$, where $P_{j}(z)=z^{3}+a_{j} z^{2}+b_{j} z+c_{j}$ is defining polynoimal of $S_{j}$ for $j=1,2$, and hence

$$
\begin{aligned}
& \left(a_{2}-a_{1}\right) f^{2} g^{2}+\left(b_{2}-b_{1}\right) f g(f+g)+\left(c_{2}-c_{1}\right)\left(f^{2}+f g+g^{2}\right) \\
& +\left(a_{1} b_{2}-a_{2} b_{1}\right) f g+\left(a_{1} c_{2}-a_{2} c_{1}\right)(f+g)+\left(b_{1} c_{2}-b_{2} c_{1}\right)=0 .
\end{aligned}
$$

Let $S_{j}=\left\{\xi_{j}\right\}$ for $j=3,4,5$. Now, assume that

$$
\begin{aligned}
& \left(a_{2}-a_{1}\right) \xi_{j}{ }^{4}+2\left(b_{2}-b_{1}\right) \xi_{j}{ }^{3}+3\left(c_{2}-c_{1}\right) \xi_{j}{ }^{2} \\
& +\left(a_{1} b_{2}-a_{2} b_{1}\right) \xi_{j}{ }^{2}+2\left(a_{1} c_{2}-a_{2} c_{1}\right) \xi_{j}+\left(b_{1} c_{2}-b_{2} c_{1}\right) \neq 0,
\end{aligned}
$$

for $j=4,5$. Then $\xi_{4}, \xi_{5}$ are exceptional values of $f$ and $g$, and hence there exist entire functions $\alpha, \beta$ without zeros such that

$$
\frac{f-\xi_{4}}{f-\xi_{5}}=\alpha, \quad \frac{g-\xi_{4}}{g-\xi_{5}}=\beta .
$$

From these we get

$$
f=\frac{\xi_{4}-\xi_{5} \alpha}{1-\alpha}, \quad g=\frac{\xi_{4}-\xi_{5} \beta}{1-\beta} .
$$

By substituting these into (7), we obtain

$$
\sum_{0 \leq j, k \leq 2} A_{j k} \alpha^{j} \beta^{k}=0
$$


where $A_{j k}$ are constants and, in particular, $A_{00}=P\left(\xi_{4}, \xi_{4}\right) \neq 0$ and $A_{22}=$ $P\left(\xi_{5}, \xi_{5}\right) \neq 0$. Here,

$$
\begin{aligned}
P(z, w) & =\left(a_{2}-a_{1}\right) z^{2} w^{2}+\left(b_{2}-b_{1}\right) z w(z+w)+\left(c_{2}-c_{1}\right)\left(z^{2}+z w+w^{2}\right) \\
& +\left(a_{1} b_{2}-a_{2} b_{1}\right) z w+\left(a_{1} c_{2}-a_{2} c_{1}\right)(z+w)+\left(b_{1} c_{2}-b_{2} c_{1}\right) .
\end{aligned}
$$

Since $f$ and $g$ are not constant, neither $\alpha$ nor $\beta$ is constant. By applying Lemma 2.2 to this equation, we can induce that one of $\alpha \beta, \alpha^{2} \beta$ and $\alpha \beta^{2}$ is constant.

If $\alpha^{2} \beta$ is constant, then

$$
\left(\frac{f-\xi_{4}}{f-\xi_{5}}\right)^{2}=c_{0} \frac{g-\xi_{5}}{g-\xi_{4}},
$$

where $c_{0}$ is a nonzero constant, and it follows from this that $T(r, g)=2 T(r, f)+$ $S(r, f)$. On the other hand, by assumption, we have

$$
\begin{aligned}
4 T(r, g) & \leq \sum_{j=1,2}\left\{\bar{N}\left(r, 1 /\left(g-\xi_{j}\right)\right)+\bar{N}\left(r, 1 /\left(g-\eta_{j}\right)\right)+\bar{N}\left(r, 1 /\left(g-\zeta_{j}\right)\right)\right\}+S(r, g) \\
& =\sum_{j=1,2}\left\{\bar{N}\left(r, 1 /\left(f-\xi_{j}\right)\right)+\bar{N}\left(r, 1 /\left(f-\eta_{j}\right)\right)+\bar{N}\left(r, 1 /\left(f-\zeta_{j}\right)\right)\right\}+S(r, g) \\
& \leq 6 T(r, f)+S(r, g)
\end{aligned}
$$

by the second fundamental theorem. However, these are not compatible. Hence $\alpha^{2} \beta$ is not constant, and similary neither is $\alpha \beta^{2}$.

Therefore $\alpha \beta$ is a constant, i.e.,

$$
\frac{f-\xi_{4}}{f-\xi_{5}} \cdot \frac{g-\xi_{4}}{g-\xi_{5}}=c_{0}
$$

where $c_{0}$ is a nonzero constant. This induces that there is a relation $f=T(g)$, where $T$ is a Möbius transformation interchanging $\xi_{4}$ and $\xi_{5}$ and it is of order 2.

Since $f$ and $g$ have two exceptional values $\xi_{4}$ and $\xi_{5}$, they have no more exceptional values, and hence $\xi_{3}$ is a fixed point of $T$. Since Möbius transformation $T$ has at most one fixed point in $S_{1} \cup S_{2}$, we may assume that it has no fixed point in $S_{1}$, and hence $E\left(\xi_{1}, \xi_{1}\right)=E\left(\eta_{1}, \eta_{1}\right)=E\left(\zeta_{1}, \zeta_{1}\right)=\emptyset$. If $E\left(\xi_{1}, \eta_{1}\right) \neq \emptyset$, then $\eta_{1}=$ $T\left(\xi_{1}\right)$. Since $T$ is one-to-one and of order 2 , we see that $E\left(\xi_{1}, \zeta_{1}\right)=E\left(\eta_{1}, \zeta_{1}\right)=\emptyset$. Therefore $\zeta_{1}$ is an exceptional value of $f$ and $g$, which is a contradiction. By the same way, we can get a contradiction in the case that $E\left(\xi_{1}, \zeta_{1}\right) \neq \emptyset$. However, these imply another contradction that $\xi_{1}$ is an exceptional value of $f$ and $g$. Hence, we conclude that $f=g$.

Theorem 3.1. Let $S_{1}, \cdots, S_{5}$ be pairwise disjoint sets in $\mathbb{C}$ with $\sharp S_{1}=\sharp S_{2}=3$ and $\sharp S_{3}=\sharp S_{4}=\sharp S_{5}=1$. Let $P_{j}(z)=z^{3}+a_{j} z^{2}+b_{j} z+c_{j}$ be defining polynomial of $S_{j}$ for $j=1,2$ and let $S_{j}=\left\{\xi_{j}\right\}$ for $j=3,4,5$. If (8) holds for at least two $j$ in $\{3,4,5\}$, then two nonconstant meromorphic functions on $\mathbb{C}$ sharing $S_{1}, \cdots, S_{5} I M$ are identical. 


\section{MEROMORPHIC FUNCTIONS SHARING THREE THREE-POINT SETS AND TWO VALUES IM}

Let $f$ and $g$ be two meromorphic functions on $\mathbb{C}$ sharing $S_{1}, \cdots, S_{5}$ IM, where $S_{1}, S_{2}, S_{3}$ are three-point sets in $\mathbb{C}$ and $S_{4}, S_{5}$ are one-point sets in $\mathbb{C}$, and $S_{1}, \cdots, S_{5}$ are pairwise disjoint. Suppose that $f \not \equiv g$. Then, by using Theorem 2.3, we may assume that $P_{1}(f) / P_{2}(f)=P_{1}(g) / P_{2}(g)$, where $P_{j}(z)=z^{3}+a_{j} z^{2}+b_{j} z+c_{j}$ is defining polynoimal of $S_{j}$ for $j=1,2,3$, and hence we have (7). Let $S_{j}=\left\{\xi_{j}\right\}$ for $j=4,5$. If we suppose that

$$
\begin{aligned}
& \left(a_{l}-a_{k}\right) \xi_{j}{ }^{4}+2\left(b_{l}-b_{k}\right) \xi_{j}{ }^{3}+3\left(c_{l}-c_{k}\right) \xi_{j}{ }^{2} \\
& +\left(a_{k} b_{l}-a_{l} b_{k}\right) \xi_{j}{ }^{2}+2\left(a_{k} c_{l}-a_{l} c_{k}\right) \xi_{j}+\left(b_{k} c_{l}-b_{l} c_{k}\right) \neq 0,
\end{aligned}
$$

holds for $j=4,5$ and distinct $k, l$ in $\{1,2,3\}$, then $\xi_{4}, \xi_{5}$ are exceptional values of $f$ and $g$, and hence, as in the $\S 3$, we can obtain a relation $f=T(g)$, where $T$ is a Möbius transformation interchanging $\xi_{4}$ and $\xi_{5}$ and it is of order 2 .

Since $T$ has at most two fixed points, we may assume that there is no fixed point in $S_{1}$. Again, by the same way in $\S 3$, we can get a contradiction, and we obatain the following theorem:

Theorem 4.1. Let $S_{1}, \cdots, S_{5}$ be pairwise disjoint sets in $\mathbb{C}$ with $\sharp S_{1}=\sharp S_{2}=$ $\sharp S_{3}=3$ and $\sharp S_{4}=\sharp S_{5}=1$. Let $P_{j}(z)=z^{3}+a_{j} z^{2}+b_{j} z+c_{j}$ be defining polynomial of $S_{j}$ for $j=1,2,3$ and let $S_{j}=\left\{\xi_{j}\right\}$ for $j=4,5$. Assume that (9) holds for distinct $k, l$ in $\{1,2,3\}$ and $j=4,5$. Then, two nonconstant meromorphic functions on $\mathbb{C}$ sharing $S_{1}, \cdots, S_{5} I M$ are identical.

\section{MEROMORPHIC FUNCTIONS SHARING FOUR THREE-POINT SETS AND A VALUE IM}

Let $f$ and $g$ be two meromorphic functions on $\mathbb{C}$ sharing $S_{1}, \cdots, S_{5}$ IM, where $S_{1}, S_{2}, S_{3}, S_{4}$ are three-point sets in $\mathbb{C}$ and $S_{5}$ is a one-point set in $\mathbb{C}$, and $S_{1}, \cdots, S_{5}$ are pairwise disjoint. Suppose that $f \not \equiv g$. Let $P_{j}(z)=z^{3}+a_{j} z^{2}+b_{j} z+c_{j}$ be defining polynomial of $S_{j}$ for $j=1,2,3,4$. Then, by using Theorem 2.3, we may assume that $P_{1}(f) / P_{2}(f)=P_{1}(g) / P_{2}(g)$, and hence we have (7). Let $S_{5}=\left\{\xi_{5}\right\}$. If we suppose that

$$
\begin{aligned}
& \left(a_{k}-a_{j}\right) \xi_{5}{ }^{4}+2\left(b_{k}-b_{j}\right) \xi_{5}{ }^{3}+3\left(c_{k}-c_{j}\right) \xi_{5}{ }^{2} \\
& +\left(a_{j} b_{k}-a_{k} b_{j}\right) \xi_{5}{ }^{2}+2\left(a_{j} c_{k}-a_{k} c_{j}\right) \xi_{5}+\left(b_{j} c_{k}-b_{k} c_{j}\right) \neq 0,
\end{aligned}
$$

for distinct $j, k$ in $\{1,2,3,4\}$, then $\xi_{5}$ is an exceptional values of $f$ and $g$.

Let $S_{j}=\left\{\xi_{j}, \eta_{j}, \zeta_{j}\right\}$ for $j=3,4$. We separate $S_{3}, S_{4}$ into two types: (A) some of $E\left(\xi_{j}, \eta_{j}\right), E\left(\xi_{j}, \zeta_{j}\right), E\left(\eta_{j}, \zeta_{j}\right)$ are not empty; (B) all of $E\left(\xi_{j}, \eta_{j}\right), E\left(\xi_{j}, \zeta_{j}\right)$, $E\left(\eta_{j}, \zeta_{j}\right)$ are empty. Then we consider three cases: (I) both of $S_{3}$ and $S_{4}$ are of 
type (B); (II) one of $S_{3}$ and $S_{4}$ is of type (B) and the other is of type (A); (III) both of $S_{3}$ and $S_{4}$ are of type (A).

The case (I). Both of $S_{3}$ and $S_{4}$ are of type (B). Then $f$ and $g$ share $\xi_{j}, \eta_{j}, \zeta_{j}$ IM for $j=3,4$. By Theorem 1.2 , we get $f=g$.

The case (II). One of $S_{3}$ and $S_{4}$ is of type (B) and the other is of type (A). We may assume that $S_{3}$ is of type (B) and $S_{4}$ is of type (A). Moreover, we may assume that $E\left(\xi_{4}, \eta_{4}\right) \neq \emptyset$. Then, we have $P_{1}\left(\xi_{4}\right) / P_{2}\left(\xi_{4}\right)=P_{1}\left(\eta_{4}\right) / P_{2}\left(\eta_{4}\right)$, and by assumption and Lemma 2.1 we have $E\left(\xi_{4}, \zeta_{4}\right)=E\left(\eta_{4}, \zeta_{4}\right)=\emptyset$, which implies that $f$ and $g$ share $\left\{\xi_{4}, \eta_{4}\right\}$ and $\left\{\zeta_{4}\right\}$ IM. Since $f$ and $g$ share also $\xi_{3}, \eta_{3}, \zeta_{3}$ and $\xi_{5}$ IM, by using Theorem 1.2, we get $f=g$.

The case (III). Both of $S_{3}$ and $S_{4}$ are of type (A). We may assume that $E\left(\xi_{j}, \eta_{j}\right) \neq \emptyset$ for $j=3,4$. Then, we have $P_{1}\left(\xi_{j}\right) / P_{2}\left(\xi_{j}\right)=P_{1}\left(\eta_{j}\right) / P_{2}\left(\eta_{j}\right)$, and by assumption and Lemma 2.1 we have $E\left(\xi_{j}, \zeta_{j}\right)=E\left(\eta_{j}, \zeta_{j}\right)=\emptyset$, which implies that $f$ and $g$ share $\left\{\xi_{j}, \eta_{j}\right\}$ and $\left\{\zeta_{j}\right\}$ IM for $j=3,4$. By using Theorem 1.3, there exists a Möbius transformation $T$ such that $f=T \circ g$.

Assume that $f \neq g$, and hence $T$ is not the identity. Then $T$ has at most two fixed points, and hence, we may assume that $E\left(\xi_{5}, \xi_{5}\right)=\emptyset$. In this case, $\xi_{5}$ is an exceptional value of $f$ and $g$. Furthermore, $T$ interchanges $\xi_{j}$ and $\eta_{j}$ for at least one of $j \in\{3,4\}$, which are also fixed points of $T^{2}=T \circ T$. Since the fixed points of $T$ are also those of $T^{2}, T^{2}$ has at least three fixed points. Therefore, $T^{2}$ is the identity, as mentioned in Remark of Theorem 1.3.

(i) The case where $E\left(\zeta_{4}, \zeta_{4}\right)=\emptyset$ or $E\left(\zeta_{3}, \zeta_{3}\right)=\emptyset$. It is enough to consider the case where $E\left(\zeta_{4}, \zeta_{4}\right)=\emptyset$. In this case $\zeta_{4}$ and $\xi_{5}$ are exceptional values of $f$ and $g$ and they have no more exceptional values. Therefore $E\left(\zeta_{3}, \zeta_{3}\right) \neq \emptyset$, and hence $\zeta_{3}$ is a fixed point $T$. Since $T$ has at most two fixed points, we may assume that all $E\left(\xi_{1}, \xi_{1}\right), E\left(\eta_{1}, \eta_{1}\right), E\left(\zeta_{1}, \zeta_{1}\right)$ are empty. Furthermore, we may assume that $E\left(\xi_{1}, \eta_{1}\right) \neq \emptyset$ since $f^{-1}\left(S_{1}\right) \neq \emptyset$. Then $T\left(\xi_{1}\right)=\eta_{1}$ and $T\left(\eta_{1}\right)=\xi_{1}$. Since $T$ is a one-to-one mapping of $\overline{\mathbb{C}}$ onto $\overline{\mathbb{C}}, E\left(\xi_{1}, \zeta_{1}\right)=\emptyset, E\left(\eta_{1}, \zeta_{1}\right)=\emptyset$. However, this implies that $\zeta_{1}$ is an exceptional value of $f$ and $g$, which is a contradiction.

(ii) The case where $E\left(\zeta_{3}, \zeta_{3}\right) \neq \emptyset$ and $E\left(\zeta_{4}, \zeta_{4}\right) \neq \emptyset$. In this case $\zeta_{3}$ and $\zeta_{4}$ are fixed points of $T$, and it has no more fixed points, in particular, $E\left(\xi_{j}, \xi_{j}\right)=$ $E\left(\eta_{j}, \eta_{j}\right)=E\left(\zeta_{j}, \zeta_{j}\right)=\emptyset$ for $j=1,2$. Since $f$ and $g$ have an exceptional value $\xi_{5}$, we may assume that they have no exceptional value in $S_{1}$. Hence we may assume that $E\left(\xi_{1}, \eta_{1}\right) \neq \emptyset$, and we can get a contradiction as in the case (i).

After all, we have $f=g$.

Theorem 5.1. Let $S_{1}, \cdots, S_{5}$ be pairwise disjoint sets in $\mathbb{C}$ with $\sharp S_{1}=\sharp S_{2}=\sharp S_{3}=$ $\sharp S_{4}=3$ and $\sharp S_{5}=1$. Let $P_{j}$ be defining polynomial of $S_{j}$ for $j=1,2,3,4$ and let $S_{5}=\left\{\xi_{5}\right\}$. Assume that $P_{1}, P_{2}, P_{3}, P_{4}$ are linearly independent over $\mathbb{C}$, and (10) holds for distinct for distinct $1 \leq j, k \leq 4$. Then, two nonconstant meromorphic functions on $\mathbb{C}$ sharing $S_{1}, \cdots, S_{5} I M$ are identical.

Acknoledgement. The authors are grateful to the referee for many helpful suggestions. 


\section{REFERENCES}

[1] F. Gross, Factorization of meromorphic functions and some open problems, "Complex Analysis, Kentucky 1976" (Proc. Conf.) Lecture Notes in Mathematics, Vol.599, pp.51-69, Springer-Verlag, Berlin, 1977.

[2] W. K. Hayman, Meromorphic functions, Clarendon Press, Oxford, 1964.

[3] S. LANG, Introduction to Complex Hyperbolic Spaces, Springer-Verlag, New York, 1987.

[4] R. Nevanlinna, Einige Eindeutigkeitssätze in der Theorie der meromorphen Funktionen, Acta Math., 48 (1926), 367-391.

[5] R. Nevanlinna, Le théorèm de Picard-Borel et la théorie des fonctions méromorphes, Gauthier-Villars, Paris, 1929

[6] T. Oкамото and M. Shirosaki, A characterization of collections of two-point sets with the uniqueness property, Tohoku Math. J., 57 (2005), 597-603.

[7] M. Shirosaki, On meromorphic functions sharing a one-point set and three two-point sets CM, Kodai Math. J., 36 (2013), 56-68.

[8] M. Shirosaki, On meromorphic functions sharing four two-point sets CM, Kodai Math. J., 36 (2013), 386-395.

[9] M. ShIRosaki, On meromorphic functions sharing five one-point sets or two two-point sets IM, Proc Japan Acad., Ser.A, 86 (2010), 6-9.

[10] M. ShIRosAKI, On meromorphic functions sharing some finite sets IM, Bull. Korean Math. Soc., 55 (2018), 865-870.

[11] M. Shirosaki And M. TAketani, On meromorphic functions sharing two one-point sets and two two-point sets, Proc Japan Acad., Ser.A, 83 (2007), 32-35.

[12] K. TohGe, Meromorphic functions covering certain finite sets at the same points, Kodai Math. J., 11 (1988), 249-279. 